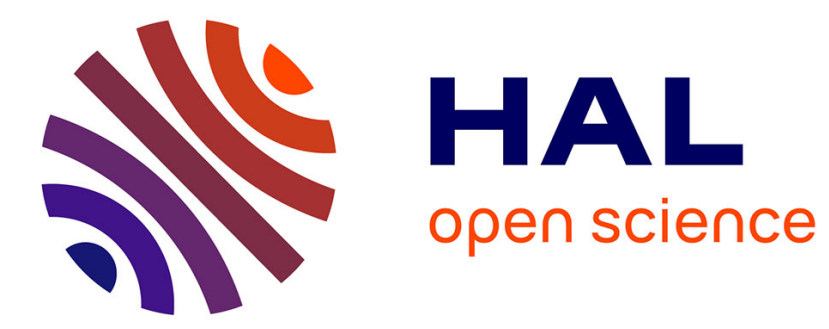

\title{
Vers la stagnation du revenu agricole
}

Michel Bourdon

\section{To cite this version:}

Michel Bourdon. Vers la stagnation du revenu agricole. Sciences Agronomiques Rennes, 1978, pp.1-7. hal-02308954

\section{HAL Id: hal-02308954 \\ https://hal.science/hal-02308954}

Submitted on 8 Oct 2019

HAL is a multi-disciplinary open access archive for the deposit and dissemination of scientific research documents, whether they are published or not. The documents may come from teaching and research institutions in France or abroad, or from public or private research centers.
L'archive ouverte pluridisciplinaire HAL, est destinée au dépôt et à la diffusion de documents scientifiques de niveau recherche, publiés ou non, émanant des établissements d'enseignement et de recherche français ou étrangers, des laboratoires publics ou privés.

\section{(이) $\$$}

Distributed under a Creative Commons Attribution - NonCommercial - NoDerivatives| 4.0 


\title{
VERS LA STAGNATION DU REVENU AGRICOLE
}

\author{
M. BOURDON
}

Chaire d'Economie Rurale - E.N.S.A. Rennes.

"Je supplie les lecteurs de ne pas verser une seule larme sur la suppression des indexations: ce n'est que la mort d'une illusion".

\section{P. FROMONT}

Chronique du Figaro Agricole, Février 1959.

Les résultats confirmés des trois dernières campagnes agricoles ne laissent pas d'être alarmants ; compte tenu de l'érosion monétaire, le revenu global de la branche agricole(1) a chaque fois baissé d'une année sur l'autre et les premières estimations provisoires dont il est fait état pour 1977 ne semblent guère très encourageantes. On peut certes, avancer que ces années de "vaches maigres" le doivent à la crise de l'énergie, à l'accélération de l'inflation et bien entendu à l'exceptionnelle sécheresse et que la paysannerie va enfin se sortir de ce mauvais pas, une fois le désordre économique disparu.

En réalité, I'habitude de voir régulièrement progresser le RBA en valeur nominale dissimule certains mécanismes spécifiques de la croissance agricole dont la dépendance vis-à-vis des moyens de production, se raffermit sans cesse. L'influence de deux paramètres de coût s'avère tellement décisive sur le rapport output/input que le revenu d'activité ne peut guère désormais espérer augmenter tout au moins en valeur réelle. Pour le démontrer, nous pouvons recourir à un modèle relativement simple de formation du revenu dont on peut vérifier l'adéquation aux réalités de longue période en utilisant notamment la série rétrospective réactualisée des nouveaux comptes de l'Agriculture Française portant sur les années 1959-1970.

(1) - Le concept actuel de RBA (Revenu Brut Agricole) qui est apparu en 1976 en Comptabilité Nationale et a remplacé l'ancien RBE (Revenu Brut d'Exploitation), représente la rémunération du travail et du capital (avant amortissement) de l'exploitant et de sa famille. 


\section{I - PRÉSENTATION DU MODELLE.}

En économie de marché, l'agrégat valeur ajoutée se définit comme la différence entre les recettes et les consommations intermédiaires : il est gouverné par la relation :

$$
V_{A}=Q_{A} P_{A}-q_{i} P_{i}
$$

dont les composantes appliquées à l'activité agricole sont :

$Q_{A}=$ quantités produites et consommées en denrées agricoles dans un certain système de prix :

$\mathrm{q}_{i}=$ quantités de facteurs extérieurs utilisés pour la production agricole :

$\mathrm{P}_{\mathrm{A}}=$ niveau relatif des prix agricoles à la production :

$p_{i}=$ niveau' relatif des prix industriels des produits nécessaires à l'agriculture.

En admettant que la marge d'exploitation demeure à peu près constante, l'évolution annuelle du revenu agricole brut (que nous appelons $R_{A}$ ), pour des taux de variation relativement faibles, obéit à l'équation :

$$
\frac{d R_{A}}{R_{A}}=\frac{d V_{A}}{V_{A}}=\frac{\left(P_{A} \cdot d Q_{A}+Q_{A} \cdot d P_{A}\right)-\left(q_{i} \cdot d p_{i}+p_{i} \cdot d q_{i}\right)}{Q_{A} \cdot P_{A}-q_{i} \cdot p_{i}}
$$

Si pour simplifier l'écriture on pose que :

$$
q_{i} \cdot p_{i}=\lambda \cdot Q_{A} \cdot P_{A}
$$

$\lambda$ représentant une certaine part des coûts dans le produit brut, alors :

$$
\frac{d R_{A}}{R_{A}}=\frac{P_{A} \cdot d Q_{A}+Q_{A} \cdot d P_{A}}{Q_{A} \cdot P_{A}(1-\lambda)}-\frac{\left(q_{i} \cdot d p_{i}+p_{i} \cdot d q_{i}\right)}{q_{i} \cdot p_{i}\left(\frac{1}{\lambda}-1\right)}
$$

Soit :

$$
\frac{d R_{A}}{R_{A}}=\frac{d Q_{A}}{Q_{A}}\left(\frac{1}{1-\lambda}\right)+\frac{d P_{A}}{P_{A}}\left(\frac{1}{1-\lambda}\right)-\frac{d p_{i}}{p_{i}}\left(\frac{1}{\frac{1-\lambda}{\lambda}}\right)-\frac{d q_{i}}{q_{i}} \frac{\left(\frac{1}{1-\lambda}\right)}{\lambda}
$$

d'où :

$$
\frac{d R_{A}}{R_{A}}=\frac{d Q_{A}}{Q_{A}}\left(\frac{1}{1-\lambda}\right)-\frac{d q_{i}}{q_{i}}\left(\frac{\lambda}{1-\lambda}\right)+\frac{d P_{A}}{P_{A}}\left(\frac{1}{1-\lambda}\right)-\frac{d p_{i}}{p_{i}}\left(\frac{\lambda}{1-\lambda}\right)
$$

Introduisons à présent le concept d'élasticité de la production qui est par définition le quotient produit facteur en termes physiques relatifs et s'écrit donc :

$$
\gamma=\frac{\frac{d Q_{A}}{Q_{A}}}{\frac{d q_{i}}{q_{i}}}
$$


Notre équation précédente devient en conséquence :

$$
\frac{d R_{A}}{R_{A}}=\frac{d Q_{A}}{Q_{A}}\left(\frac{1}{1-\lambda}\right)-\frac{d Q_{A}}{Q_{A}} \times \frac{1}{\gamma}\left(\frac{\lambda}{1-\lambda}\right)
$$

et en définitive :

$$
\frac{d R_{A}}{R_{A}}=\frac{d Q_{A}}{Q_{A}}\left[\frac{\gamma-\lambda}{\gamma(1-\lambda)}\right]
$$

On peut donc valablement considérer qu'à rapports de prix stables entre l'agriculture et ses fournisseurs, le taux de croissance du Revenu Agricole est pour l'essentiel tributaire de la progression de la production affectée d'un certain coefficient qui résulte de la combinaison de la structure des coûts et de l'élasticité de la production ; or ces deux variables évoluent, sinon au même rythme, de manière symétriquement inverse : $\gamma$ diminue pendant que $\lambda$ augmente si bien que le coefficient d'ensemble décroît, tend à long terme vers zéro et devient même négatif au-delà d'une certaine limite. Il s'ensuit au total une action de plus en plus faible de l'expansion de la production sur la hausse du revenu de l'agriculture.

$\mathrm{Si}$ à titre indicatif on désire chiffrer la valeur du multiplicateur global en effectuant les calculs par tranche

\begin{tabular}{|c|c|c|c|c|c|c|c|}
\hline & 0,35 & 0.40 & 0,45 & 0,50 & 0,55 & 0,60 & 0,65 \\
\hline 0,45 & 0,341880 & 0,185185 & 0 & $-0,222222$ & $-0,493827$ & $-0,833333$ & $-1,269841$ \\
\hline 0,50 & 0,461538 & 0,333333 & 0,181818 & 0 & $-0,222222$ & $-0,500000$ & $-0,857142$ \\
\hline 0,55 & 0,559440 & 0,454545 & 0,330578 & 0,181818 & 0 & $-0,227272$ & $-0,519480$ \\
\hline 0,60 & 0,641025 & 0,555555 & 0,454545 & 0,333333 & 0,185185 & 0 & $-0,238095$ \\
\hline 0,65 & 0,710059 & 0,641025 & 0,559440 & 0,461538 & 0,341880 & 0,192307 & 0 \\
\hline 0,70 & 0.769230 & 0,714285 & 0,649350 & 0,571428 & 0,476190 & 0,357142 & 0,204081 \\
\hline 0,75 & 0,820512 & 0,777777 & 0,727272 & 0,666666 & 0,592592 & 0,500000 & 0,380952 \\
\hline
\end{tabular}
de 0,05 dans une fourchette de valeurs observées ou prévisibles pour $\gamma$ et $\lambda$ on obtient l'abaque suivant :

On s'aperçoit aisément, en particulier dans la zone délimitée, que le coefficient obtenu exerce un effet réducteur très net puisqu'il est inférieur à 0,20 et l'on comprend combien l'élévation du revenu agricole ne peut qu'être minime en dépit d'une croissance mème satisfaisante de la production. Essayons maintenant de voir si notre modèle s'adapte bien aux données constatées dans les Comptes Nationaux. 


\section{II - APPLICATION STATISTIQUE.}

En utilisant les matériaux appropriés ${ }^{(1)}$ et en calculant quelques ratios significatifs, tentons de chiffrer les deux paramètres-clés puis testons-en la conformité au réel.

Depuis 1960 la part des approvisionnements dans la valeur de l'output agricole s'établit comme suit :

Tableau 1

\begin{tabular}{|c|c|c|c|}
\hline Années & $\begin{array}{c}\text { Production totale de la } \\
\text { branche (en millions de } \\
\text { francs courants) }\end{array}$ & $\begin{array}{c}\text { Consommations inter- } \\
\text { médiaires (en millions } \\
\text { de francs courants) }\end{array}$ & $\begin{array}{c}\text { Consommations } \\
\text { intermédiaires } \\
\text { en \% de la production }\end{array}$ \\
\hline 1960 & 48247 & 18138 & $37,59 \%$ \\
1961 & 47406 & 18558 & $39,14 \%$ \\
1962 & 55269 & 21532 & $38,95 \%$ \\
1963 & 57426 & 22889 & $39,85 \%$ \\
1964 & 56605 & 22973 & $40,58 \%$ \\
1965 & 61296 & 25213 & $41,13 \%$ \\
1966 & 64561 & 27101 & $41,97 \%$ \\
1967 & 68778 & 28770 & $41,83 \%$ \\
1968 & 70079 & 29782 & $42,49 \%$ \\
1969 & 72623 & 29782 & $41,00 \%$ \\
1970 & 79773 & 32963 & $41,32 \%$ \\
1971 & 86006 & 36396 & $42,31 \%$ \\
1972 & 99750 & 40253 & $40,35 \%$ \\
1973 & 118218 & 48180 & $40,75 \%$ \\
1974 & 124660 & 58622 & $47,02 \%$ \\
1975 & 128957 & 60693 & $47,06 \%$ \\
1976 & 145253 & 71304 & $49,09 \%$ \\
& & & \\
\hline
\end{tabular}

Malgré une évolution quelque peu irrégulière on constate la part sans cesse croissante prise par les dépenses courantes d'exploitation dans les recettes brutes : la valeur de $\lambda$ pourrait ainsi aujourd'hui se situer aux alen. tours de 0,45 en année normale.

Afin d'estimer l'élasticité de l'appareil productif reprenons maintenant les mêmes données non plus en valeurs mais cette fois-ci en quantités physiques ; les Comptes de l'Agriculture nous donnent, chaque année par rapport à la précédente, leur croissance en volume que l'on peut convertir en progression indiciaire' sur la base $1959=100$.

(1) - Tous les chiffres de cette étude, non calculés par l'auteur, sont extraits des Comptes de l'Agriculture, série historique et années récentes : Collection de I'INSEE, série $\mathrm{C}, \mathrm{n}^{\circ} 48$; ainsi que Ministère de I'Agriculture. SCEES. Collection de statistique agricole, étude $n^{\circ} 152$, Juillet 1977. 
Tableau 2

\begin{tabular}{|c|c|c|c|c|}
\hline \multirow{2}{*}{ Années } & \multicolumn{2}{|c|}{ Production totale } & \multicolumn{2}{|c|}{ Consommations intermédiaires } \\
\hline & Taux annuel & Indice & Taux annuel & Indice \\
\hline 1960 & $14,9 \%$ & 114,9 & $10,2 \%$ & 110,2 \\
\hline 1961 & $-\quad 3,8 \%$ & 110,5 & $0,2 \%$ & 110,4 \\
\hline 1962 & $5,9 \%$ & 117,1 & $4,0 \%$ & 114,8 \\
\hline 1963 & $1,3 \%$ & 118,6 & $9,4 \%$ & 125,6 \\
\hline 1964 & $0,2 \%$ & 118,8 & $0,3 \%$ & 126,0 \\
\hline 1965 & $6,3 \%$ & 126,3 & $7,6 \%$ & 135,6 \\
\hline 1966 & $0,6 \%$ & 127,1 & $4,6 \%$ & 141,8 \\
\hline 1967 & $6,2 \%$ & 134,9 & $4,9 \%$ & 148,8 \\
\hline 1968 & $2,2 \%$ & 137,9 & $2,0 \%$ & 151,7 \\
\hline 1969 & $-\quad 3,5 \%$ & 133,1 & $-\quad 0,9 \%$ & 150,4 \\
\hline 1970 & $3,6 \%$ & 137,9 & $5,0 \%$ & 157,9 \\
\hline 1971 & $3,5 \%$ & 142,7 & $5,8 \%$ & 167,0 \\
\hline 1972 & $2,4 \%$ & 146,1 & $4,9 \%$ & 175,2 \\
\hline 1973 & $7,5 \%$ & 157,1 & $9,1 \%$ & 191,2 \\
\hline 1974 & $-\quad 0,8 \%$ & 155,8 & $-\quad 0,5 \%$ & 190,2 \\
\hline 1975 & $-\quad 4,5 \%$ & 148,8 & $-\quad 4,0 \%$ & 182,6 \\
\hline 1976 & $-\quad 1,8 \%$ & 146,1 & $4,1 \%$ & 190,1 \\
\hline
\end{tabular}

II serait de peu d'utilité de calculer pour la série entière, l'élasticité instantanée, c'est-à-dire d'une année sur l'autre : il est préférable de connaître la valeur prise par $\gamma$ à moyen ou long terme, les rapports de variation pouvant alors être établis en choisissant quelques jalons; nos évaluations opérées sur une moyenne de deux ou trois ans selon le mode de calcul apparaissent ci-dessous :

Tableau 3

\begin{tabular}{|c|c|c|c|c|c|c|c|}
\hline $\begin{array}{l}\text { Valeur du } \\
\text { Coefficient }\end{array}$ & $\begin{array}{l}1964 \\
1965\end{array}$ & $\begin{array}{c}1965 \\
1966-1967\end{array}$ & $\begin{array}{l}1967 \\
1968\end{array}$ & $\begin{array}{c}1970 \\
1971-1972\end{array}$ & $\begin{array}{l}1971 \\
1972\end{array}$ & $\begin{array}{c}1973 \\
1974-1975\end{array}$ & $\begin{array}{l}1975 \\
1976\end{array}$ \\
\hline $\begin{array}{l}\text { à long terme } \\
\text { à moyen terme }\end{array}$ & $\begin{array}{c}0,732 \\
-\end{array}$ & $\begin{array}{c}- \\
0,766\end{array}$ & $\begin{array}{c}0,724 \\
-\end{array}$ & $\begin{array}{c}- \\
0,605\end{array}$ & $\begin{array}{c}0,624 \\
-\end{array}$ & $\begin{array}{c}- \\
0,478\end{array}$ & $\begin{array}{c}0,549 \\
-\end{array}$ \\
\hline
\end{tabular}

II est incontestable que l'élasticité connait un déclin tendanciel, le coefficient actuel à retenir étant de l'ordre d'un demi-point. Constatons au passage que la plage de variation observée correspond bien aux limites de notre abaque et qu'en fonction des estimations raisonnables du couple $\lambda-\gamma(0,45-0,50)$ on évolue dans la zone déjà repérée : II faut en conséquence $s^{\prime}$ attendre à un très faible effet de croissance du revenu agricole mais voyons alors si la mesure directe du RBA aboutit à des résultats concordants. 
La valeur nominale du Revenu Agricole appréhendé ici exclusivement du point de vue économique, c'est-à-dire hors transfers sociaux, se lit directement dans les comptes nationaux. En utilisant comme déflateur l'indice obtenu à partir de la croissance annuelle des prix du Produit Intérieur Brut, pratique courante des experts de l'INSEE, on déduit naturellement la valeur réelle du RBA indépendante du rythme d'inflation :

Tableau 4

\begin{tabular}{|c|c|c|c|c|}
\hline Années & $\begin{array}{c}\text { Revenu agricole brut } \\
\text { (en millions de } \\
\text { francs courants) }\end{array}$ & $\begin{array}{c}\text { Taux annuel de } \\
\text { hausse du niveau } \\
\text { général des prix }\end{array}$ & $\begin{array}{c}\text { Indice } \\
\text { Déflateur } \\
\text { base 100 en 1959 }\end{array}$ & $\begin{array}{c}\text { Revenu agricole brut } \\
\text { (en millions de } \\
\text { francs constants) }\end{array}$ \\
\hline 1960 & 22216 & $3,0 \%$ & 103 & 21569 \\
1961 & 22911 & $2,8 \%$ & 105,9 & 21638 \\
1962 & 25299 & $4,0 \%$ & 110,1 & 22974 \\
1963 & 27952 & $5,3 \%$ & 115,9 & 24105 \\
1964 & 26349 & $3,7 \%$ & 120,2 & 21912 \\
1965 & 27473 & $2,4 \%$ & 123,1 & 22312 \\
1966 & 28552 & $2,8 \%$ & 126,6 & 22556 \\
1967 & 30055 & $2,7 \%$ & 130,0 & 23119 \\
1968 & 30545 & $4,1 \%$ & 135,3 & 22571 \\
1969 & 33382 & $6,6 \%$ & 144,2 & 23140 \\
1970 & 34808 & $5,1 \%$ & 151,6 & 22958 \\
1971 & 38098 & $5,6 \%$ & 160,1 & 23795 \\
1972 & 44863 & $6,2 \%$ & 170,0 & 26385 \\
1973 & 48980 & $7,3 \%$ & 182,4 & 26847 \\
1974 & 49984 & $10,8 \%$ & 202,1 & 24726 \\
1975 & 53337 & $12,2 \%$ & 226,8 & 23516 \\
1976 & 56413 & $9,4 \%$ & 248,1 & 22735 \\
& & & & \\
\hline
\end{tabular}

Au vu de l'ensemble des chiffres de la dernière colonne on ne peut qu'être frappé, en dépit de quelques sautes accidentelles, par la stabilité du Revenu exprimé en francs 59. On note certes que les deux années 72 et 73 s'écartent nettement du "trend" car elles ont bénéficié d'une conjoncture extrêmement favorable de bonnes récoltes et de prix européens revalorisés, mais ces deux "exceptions" sont rapidement annulées par les deux années suivantes qui ont été au contraire très néfastes du point de vue du revenu en raison d'un renchérissement considérable des coûts. La dernière année est caractéristique de la série puisque le RBA, en francs constants, retrouve le niveau tendanciel qu'il a connu de 1960 à 1971 compris, la moyenne de ces douze années ressortissant à $22623 \mathrm{~F}$. Ce retour à la "normale" est d'ailleurs confirmé par les premiers résultats publiés pour $1977^{(1)}$ lesquels prévoient un RBA de 61674 millions de francs courants ce qui, compte-tenu d'un taux d'inflation de $9,1 \%$ prolongerait notre série par le chiffre de $22782 \mathrm{~F}$. : en valeur réelle l'agriculture française obtient de nos jours le même revenu qu'il $y$ a quinze ans !

(1) - Cf. Economie et Statistique, n94, Novembre 1977 ; également Revue des Chambres d’agriculture, nº17-618, Décembre, 1977. 
Les seuls éléments qui viennent annuellement perturber quelque peu la régularité sérielle s'expliquent aisément par l'influence à court terme déformante des prix relatifs. Afin de ne pas surcharger le texte nous n'avons pas fait figurer les statistiques de prix, néanmoins le lecteur doit savoir que sur base 100 en 59 , les prix agricoles à la production sont fin 1976, à l'indice 233,2, cependant que ceux des inputs se situent à 232,1 : le parallélisme dans l'évolution à long terme des rapports de prix n'est-il pas étonnant ?

Un dernier mot pour corroborer la validité de notre modèle lequel supposait initialement, rappelons.le, que revenu et valeur ajoutée évoluaient à allure identique ce qui revenait implicitement à admettre le maintien du rapport RBA/VA; si en reprenant les données en francs courants on effectue le quotient entre le numérateur (tabl. 4) et le dénominateur (la valeur ajoutée étant la différence entre les deux chiffres du tabl. 1), on obtient les pourcentages suivants :

Tableau 5

\begin{tabular}{|c|c|c|c|c|c|c|c|c|}
\hline 1960 & 1961 & 1962 & 1963 & 1964 & 1965 & 1966 & 1967 & 1968 \\
\hline $73,78 \%$ & $79,41 \%$ & $74,98 \%$ & $80,93 \%$ & $78,34 \%$ & $76,13 \%$ & $76,22 \%$ & $75,12 \%$ & $75,76 \%$ \\
\hline 1969 & 1970 & 1971 & 1972 & 1973 & 1974 & 1975 & 1976 & \\
\hline $77,92 \%$ & $74,36 \%$ & $76,79 \%$ & $75,39 \%$ & $69,93 \%$ & $75,69 \%$ & $78,13 \%$ & $76,29 \%$ & \\
\hline
\end{tabular}

On se doit, là encore, de remarquer l'extraordinaire stabilité de la marge bénéficiaire nette d'exploitation qui demeure à peu près constante autour de 76 à $77 \%$; la seule exception notable est 1973 qui s'explique par une réduction drastique cette année là des subventions d'exploitation habituellement versées aux agriculteurs.

\section{CONCLUSION.}

L'illustration économétrique a révélé le bien-fondé de notre outil d'analyse. Un assujettissement de plus en plus marqué vis-à-vis d'inputs industriels, un amenuisement de l'élasticité de l'appareil de production, des rendements moins performants sont des phénomènes qui semblent désormais irréversibles en agriculture et qui sont d'ailleurs dans la logique du déclin relatif de la branche au sein de l'économie nationale. Il ne faut donc pas s'étonner de la quasi-stagnation réelle du Revenu Agricole. Profitons-en pour affirmer, contrairement à l'opinion du Professeur MARCZEWSKI (1), qu'il nous paraît présomptueux d'accuser le secteur primaire d'être à la source d'un "cercle inflationniste agricole" ; l'agriculture est un secteur fragile et dominé qui, avec peine, parvient à ménager ses marges d'exploitation ; on ne saurait blâmer les agriculteurs de se comporter en agents responsables de l'inflation au moment où leur action ne tend qu'à éviter une trop forte détérioration de leur revenu réel qui demeure constamment précaire.

(1) - J. MARCZEWSKI, Inflation et chómage en France, 1977 - Editions Economica. 


$$
\text { . }
$$

